\title{
Accuracy Issues of Discrete Hilbert Transform in Identification of Instantaneous Parameters of Vibration Signals
}

\author{
M. MEISSNER \\ Institute of Fundamental Technological Research of the Polish Academy of Sciences \\ A. Pawińskiego 5B, 02-106 Warsaw, Poland
}

\begin{abstract}
In the paper, an accuracy of the discrete Hilbert transform method in a detection of amplitude and frequency of vibration signals has been examined. This issue was studied for sinusoidal damped vibrations often encountered in practical applications. It has been shown that an inexactness of the Hilbert transform generates fictitious oscillations of amplitude and frequency. For small damping of vibrations these oscillations were significant only at both ends of a discrete signal. An error induced by a numerical algorithm was especially severe for strongly damped vibrations because it manifested itself by a large overestimation of amplitude and erroneous jumps of frequency. To reduce the error generated by the Hilbert transform method, the appropriate modification of discrete signal was proposed.
\end{abstract}

PACS: $02.30 . \mathrm{Uu}$, 46.40.Ff, 43.20.Ks

\section{Introduction}

The Hilbert transform and the associated concept of analytic signal representation, introduced by Gabor [1], have been widely adopted in signal analysis and were important tools in vibration-based machine fault diagnosis [2] as well as in many other practical applications [3]. In room acoustics, this transform was used in low-frequency range, where modal vibrations generated in enclosures are well separated, and it is employed to calculate an envelope of decaying acoustic pressure $[4,5]$. It allows to predict decay times more accurately, which is especially important for room systems producing a non-exponential sound decay [6-8]. As was shown in Ref. [4], the Hilbert transform method gives meaningful numerical data only for restrictive class of decaying vibration signals. The accurate results are obtained for small damping of vibrations because in this case a product of exponentially decaying signal and a harmonic signal satisfies approximately the Bedrosian identity [9]. In practice, real signals have a finite length, therefore, they are processed by the discrete Hilbert transform resulting in an additional inaccuracy of the numerical method because of the end effect and the time discretization [10].

The paper is organized as follows. In the first part, the most important properties of the Hilbert transform method are shortly discussed. Then, the study is focused on accuracy issues of this methodology in a detection of amplitude and frequency for free damped vibrations. Finally, a simple procedure of signal modification is proposed to reduce an enormous error of the method for strongly damped vibrations.

\section{Theoretical background}

The Hilbert transform plays an important role in a signal analysis because it can be used for a direct examination of instantaneous amplitude and frequency of vibration signals. In the continuous time domain, the Hilbert transform $\mathcal{H}$ of the real signal $x(t)$ is defined as [11]:

$$
\mathcal{H}[x(t)]=\frac{1}{\pi} \int_{-\infty}^{\infty} \frac{x(\tau)}{t-\tau} \mathrm{d} \tau,
$$

where the integral is considered as a Cauchy principal value because of the possible singularity at $\tau=t$. In practice, signals which can be analysed via the Hilbert transform are of finite length and digitally sampled, thus having the signal $x(t)$ defined in the time interval $[0, T]$ and using the uniform sampling with the period $T_{\mathrm{s}}$, we obtain the discrete-time signal $x[n]$ :

$$
x[n]=x\left(n T_{\mathrm{s}}\right), \quad n=0,1, \ldots, N .
$$

In discrete time case, the Hilbert transform $\mathcal{H}$ is replaced by the discrete Hilbert transform $\mathcal{H}_{\mathrm{d}}$ which for non-periodic discrete signals is determined by [12]:

$$
\mathcal{H}_{\mathrm{d}}\{x[n]\}= \begin{cases}\frac{2}{\pi} \sum_{m=\text { odd }} \frac{x[m]}{n-m}, & n \text { even, } \\ \frac{2}{\pi} \sum_{m=\text { even }} \frac{x[m]}{n-m}, & n \text { odd } .\end{cases}
$$

The signal $x[n]$ and its Hilbert transform $\mathcal{H}_{\mathrm{d}}\{x[n]\}$ are related to each other in such a way that they together create the so-called analytic discrete-time signal $z[n]$ defined as:

$$
z[n]=x[n]+\mathrm{j} \mathcal{H}_{\mathrm{d}}\{x[n]\}=A[n] \mathrm{e}^{\mathrm{j} \phi[n]},
$$




$$
\begin{aligned}
& A[n]=\sqrt{x^{2}[n]+\mathcal{H}_{\mathrm{d}}^{2}\{x[n]\}}, \\
& \phi[n]=\tan ^{-1}\left(\frac{\mathcal{H}_{\mathrm{d}}\{x[n]\}}{x[n]}\right),
\end{aligned}
$$

where $A[n]$ and $\phi[n]$ are the instantaneous amplitude and phase of the signal $x[n]$, respectively. In discrete time, the instantaneous frequency $\omega[n]$ of signal is a derivative of the phase $\phi[n]$ with respect to $n$, thus denoting this derivative as the prime we obtain

$$
\omega[n]=\phi^{\prime}[n]=\frac{x[n] \mathcal{H}_{\mathrm{d}}^{\prime}\{x[n]\}-x^{\prime}[n] \mathcal{H}_{\mathrm{d}}\{x[n]\}}{A^{2}[n]} .
$$

The above way of determining the instantaneous amplitude and frequency of a signal via the Hilbert transform is called the analytic signal method.

In engineering analysis, the analytic representation of discrete signals has been found very useful for many types of signals, especially for the amplitude-modulated ones, modelled as a product of two functions. A simple method for computing such a product was found by Bedrosian [9]. If two functions, say $x_{1}[n]$ and $x_{2}[n]$, represent discrete signals with non-overlapping spectra and the spectrum of $x_{1}[n]$ is lower than that of $x_{2}[n]$, then

$$
\mathcal{H}_{\mathrm{d}}\left\{x_{1}[n] x_{2}[n]\right\}=x_{1}[n] \mathcal{H}_{\mathrm{d}}\left\{x_{2}[n]\right\},
$$

thus, to compute the Hilbert transform of the product of a low-pass signal with a high-pass signal, only the high-pass signal needs to be transformed. This result is known in the literature as the Bedrosian theorem and the formula (8) is called the Bedrosian identity.

\section{Application of Hilbert transform method to free damped vibrations}

Free damped vibrations are encountered in many mechanical and structural applications such as mechanisms, machines, buildings and bridges. In acoustics, the excitation of this kind of vibrations takes place during processes of a sound build-up and a sound decay in rooms [13]. The elementary mechanical system, which is capable to free damped vibrations, is modelled by the mass-spring circuit with added damper that simulates a viscous dissipation [14]. In such a system, the oscillatory displacement of the mass is determined by

$$
\begin{aligned}
& x(t)=\mathrm{e}^{-\eta \Omega_{0} t}\left[\frac{x^{\prime}(0)+\eta \Omega_{0} x(0)}{\omega_{0}} \sin \left(\omega_{0} t\right)\right. \\
& \left.\quad+x(0) \cos \left(\omega_{0} t\right)\right],
\end{aligned}
$$

where $\eta$ is the damping ratio, $\Omega_{0}$ is the natural frequency, $\omega_{0}=\Omega_{0} \sqrt{1-\eta^{2}}$ is the natural frequency for damped vibrations, $x(0)$ and $x^{\prime}(0)$ represent initial conditions. For many practical cases the damping ratio $\eta$ is relatively small, hence the difference between natural frequencies for damped and undamped vibrations is negligible. The accuracy of the Hilbert transform method was examined for the simplest case of free damped vibration, that is when the initial displacement $x(0)$ is set to zero. Thus, a simulated discrete signal has the form

$$
s[n]=A_{0}[n] \sin \left(\omega_{0} t[n]\right),
$$

where $A_{0}[n]=\mathrm{e}^{-\eta \Omega_{0} t[n]}$ is the signal amplitude, $t[n]=$ $n T_{\mathrm{s}}$ is the discrete time and $n=0,1, \ldots, N$. The numerical tests were performed for the natural frequency $\Omega_{0}$ of $200 \mathrm{rad} / \mathrm{s}$, the sampling period $T_{\mathrm{s}}$ of $2.5 \times 10^{-4} \mathrm{~s}$, $N=2 \times 10^{4}$ and the damping ratio $\eta$ possessing two values: $5 \times 10^{-4}$ and 0.01 , corresponding to the cases of small and large damping of vibrations. Shapes of simulated discrete signal are shown in Fig. 1.
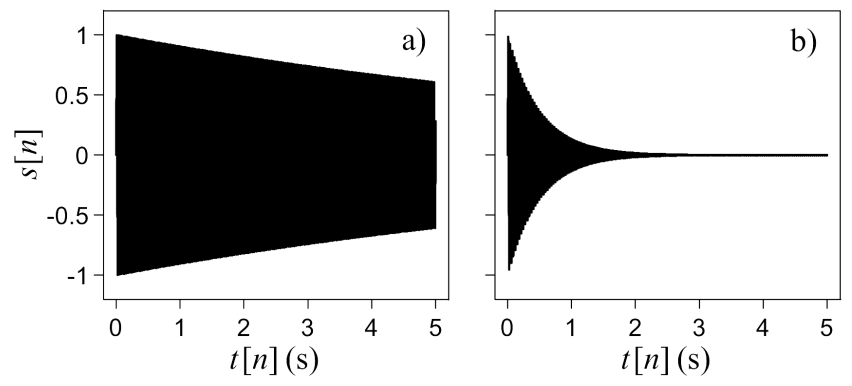

Fig. 1. Shapes of simulated discrete signal for damping ratio $\eta$ : (a) $5 \times 10^{-4}$, (b) 0.01 .

In general, errors in calculating the discrete Hilbert transform of the signal $s[n]$ are due to three main reasons. The first one is the end effect resulting from a finite duration of the signal. The second one is a time discretization through the sampling process. The third one stems from the fact that the Bedrosian identity (8) for the signal $s[n]$ is not satisfied because the exponential function has an unbounded spectrum. Thus, using a general error model, a formula for the Hilbert transform of the signal $s[n]$ can be written as

$$
\mathcal{H}_{\mathrm{d}}\{s[n]\}=\left(1+\epsilon_{1}[n]\right) A_{0}[n] \cos \left(\omega_{0} t[n]\right)+\epsilon_{2}[n],
$$

where $\epsilon_{1}[n]$ and $\epsilon_{2}[n]$ are the multiplicative and additive errors, respectively. As may be noted, the additive term represents non-oscillating component of the error. Substituting Eq. (11) into Eqs. (5) and (7) it is easy to find that errors in calculating the discrete Hilbert transform cause erroneous oscillations of instantaneous amplitude and frequency. It is confirmed by calculation data depicted in Fig. 2a, b showing substantial oscillations of the amplitude ratio $A[n] / A_{0}[n]$ and the non-dimensional frequency $\omega[n] / \omega_{0}$ near lower and upper limits of a simulation time interval for the damping ratio $\eta=5 \times 10^{-4}$. Therefore, a finite time duration of a signal considerably affects a quality of a reconstruction of instantaneous signal parameters even at a small damping of vibrations. An inexactness of the analytic signal method in the whole time interval is better illustrated by the relative errors, which due to oscillations of amplitude and frequency, are defined as follows:

$$
\Delta_{1}[n]=\mathcal{E}\left|\frac{A[n]-A_{0}[n]}{A_{0}[n]}\right|, \Delta_{2}[n]=\mathcal{E}\left|\frac{\omega[n]-\omega_{0}}{\omega_{0}}\right|,
$$


where $\mathcal{E}(\cdot)$ denotes the upper envelope of a positive oscillatory function describing a relative error in a considered time interval. Calculation results shown in Fig. 2c, $d$ indicate that for the damping ratio $\eta$ equal to $5 \times 10^{-4}$ the errors $\Delta_{1}[n]$ and $\Delta_{2}[n]$ are very close in value. In the middle of time interval both of these errors are relatively small, the order of 0.001 , but they grow rapidly to values close to unity at ends of a discrete signal.

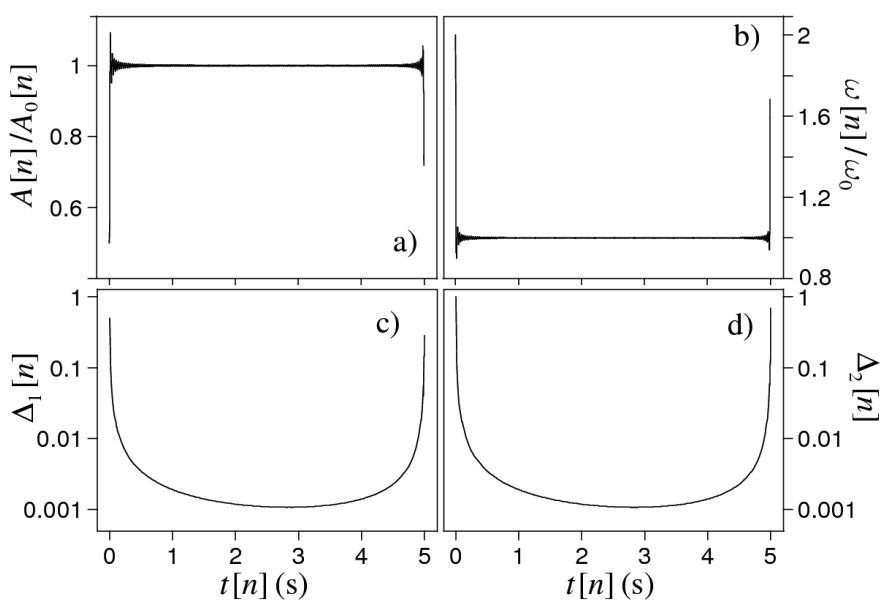

Fig. 2. Calculation results for damping ratio $\eta=$ $5 \times 10^{-4}$ : (a) amplitude ratio $A[n] / A_{0}[n]$, (b) non-dimensional frequency $\omega[n] / \omega_{0}$ and $(\mathrm{c}, \mathrm{d})$ relative errors $\Delta_{1}[n]$ and $\Delta_{2}[n]$ for signal $s[n]$.

An exactness of a numerical algorithm in a detection of amplitude and frequency can be improved by calculating $A[n]$ and $\omega[n]$ for the extended discrete signal $s_{\mathrm{e}}[n]$ given by

$$
s_{\mathrm{e}}[n]=\mathrm{e}^{-\eta \Omega_{0}|t[n]|} \sin \left(\omega_{0} t[n]\right),
$$

where $t[n]=(n-N) T_{\mathrm{S}}$ and $n=0,1, \ldots, 2 N$. Thus, the new discrete signal is determined in a double-extended time interval and represents a discrete function having a rotational symmetry with respect to the origin of coordinate system. As shown in Fig. 3a, b, the proposed signal modification significantly reduces erroneous oscillations of amplitude and frequency. This is particularly evident when comparing the errors $\Delta_{1}[n]$ and $\Delta_{2}[n]$ obtained for discrete signals $s[n]$ and $s_{\mathrm{e}}[n]$ because it appears that this modification causes about a thousandfold reduction of these errors at the lower limit of a time interval (Fig. 3c, d).

Calculation results obtained for a large damping of vibrations are depicted in Fig. 4. From these data it follows that in this case the method of calculating the amplitude and the frequency via the Hilbert transform completely fails. Now fictitious oscillations generated by a numerical procedure are so large that they cause a total deformation of the signal amplitude at ends of a discrete signal and abnormal positive and negative frequency jumps (Fig. 4a, b). Fortunately, as before, the inexactness of amplitude and frequency predictions can be greatly reduced by applying the proposed modification of a dis-

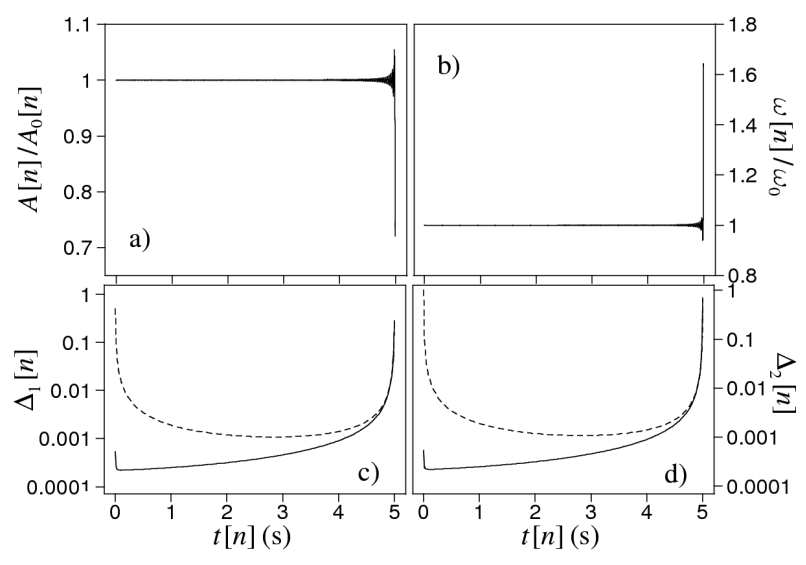

Fig. 3. Calculation results for damping ratio $\eta=$ $5 \times 10^{-4}$ : (a) amplitude ratio $A[n] / A_{0}[n]$ and (b) non-dimensional frequency $\omega[n] / \omega_{0}$ for extended signal $s_{\mathrm{e}}[n],(\mathrm{c}, \mathrm{d})$ relative errors $\Delta_{1}[n]$ and $\Delta_{2}[n]$ for signal $s[n]$ (dashed lines) and extended signal $s_{\mathrm{e}}[n]$ (solid lines).

crete signal (Fig. 4c,d). However, in this case the greatest reduction of the relative errors $\Delta_{1}[n]$ and $\Delta_{2}[n]$ are observed in the middle of a time interval (Fig. 4e,f).

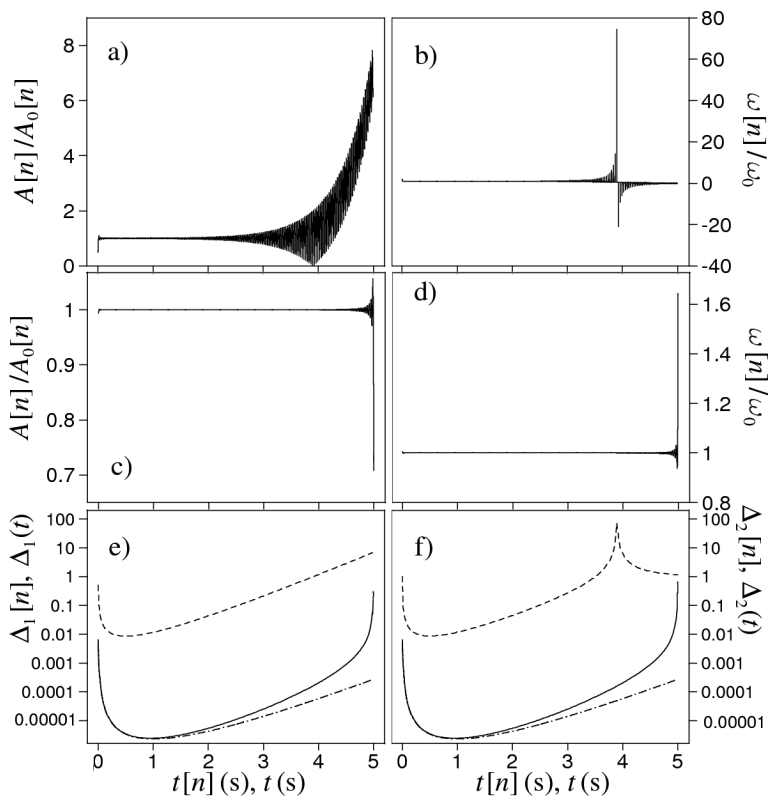

Fig. 4. Calculation results for damping ratio $\eta=$ 0.01: (a,c) amplitude ratio $A[n] / A_{0}[n]$ and $(\mathrm{b}, \mathrm{d})$ non-dimensional frequency $\omega[n] / \omega_{0}$ for signals $s[n]$ and $s_{\mathrm{e}}[n],(\mathrm{e}, \mathrm{f})$ relative errors $\Delta_{1}[n]$ and $\Delta_{2}[n]$ for signal $s[n]$ (dashed lines) and signal $s_{\mathrm{e}}[n]$ (solid lines), relative errors $\Delta_{1}(t)$ and $\Delta_{2}(t)$ calculated from Eqs. (20) and (21) (dashed-dotted lines).

The error produced by the Hilbert transform method due to an unbounded spectrum of the exponential function can be determined exactly in the continuous time domain. In this case a signal equivalent to the extended discrete signal $s_{\mathrm{e}}[n]$ is of the form 


$$
s_{\mathrm{e}}(t)=\mathrm{e}^{-\eta \Omega_{0}|t|} \sin \left(\omega_{0} t\right) .
$$

According to Ref. [4], the Hilbert transform of this signal is determined by

$$
\begin{aligned}
& \mathcal{H}\left[s_{\mathrm{e}}(t)\right]=-\mathrm{e}^{-\eta \Omega_{0}|t|} \cos \left(\omega_{0} t\right)+f(t), \\
& f(t)=\frac{4 \eta \sqrt{1-\eta^{2}}}{\pi} \int_{0}^{\infty} \frac{s \mathrm{e}^{-s \Omega_{0}|t|} \mathrm{d} s}{\left(s^{2}+2 \eta s+1\right)\left(s^{2}-2 \eta s+1\right)} .
\end{aligned}
$$

Since an application of the Hilbert transform to the pure sinusoidal signal gives [11]:

$$
\mathcal{H}\left[\sin \left(\omega_{0} t\right)\right]=-\cos \left(\omega_{0} t\right),
$$

the function $f(t)$ represents the error in a numerical method caused by the fact that spectra of signals in a product on the right side of Eq. (14) overlap. Using the analytic signal method it is easy to find that the amplitude and frequency of the signal $s_{\mathrm{e}}(t)$ are given by

$$
\begin{aligned}
& A(t) / A_{0}(t)=\sqrt{1-2 \epsilon(t) \cos \left(\omega_{0} t\right)+\epsilon^{2}(t)} \\
& \omega(t) / \omega_{0}= \\
& \frac{1+\xi(t) \sin \left(\omega_{0} t\right) / \omega_{0}-\epsilon(t)\left[\cos \left(\omega_{0} t\right)-\eta \Omega_{0} \sin \left(\omega_{0} t\right) / \omega_{0}\right]}{1-2 \epsilon(t) \cos \left(\omega_{0} t\right)+\epsilon^{2}(t)},
\end{aligned}
$$

where $A_{0}(t)=\mathrm{e}^{-\eta \Omega_{0}|t|}, \epsilon(t)=f(t) / A_{0}(t)$ and $\xi(t)=$ $f^{\prime}(t) / A_{0}(t)$. As it results from these equations, the error described by the function $f(t)$ leads to erroneous oscillations of amplitude and frequency. Since this function is positive for the damping ratio $\eta<1$, the relative errors corresponding to the maximal inaccuracy of the method are determined by

$$
\begin{aligned}
& \Delta_{1}(t)=\mathcal{E}\left|\frac{A(t)-A_{0}(t)}{A_{0}(t)}\right|=\epsilon(t), \\
& \Delta_{2}(t)=\mathcal{E}\left|\frac{\omega(t)-\omega_{0}}{\omega_{0}}\right|=\frac{\epsilon(t)}{1+\epsilon(t)},
\end{aligned}
$$

thus for $\epsilon(t)$ much smaller than unity values of $\Delta_{1}(t)$ and $\Delta_{2}(t)$ are almost identical.

In Fig. 4e, f the errors $\Delta_{1}(t)$ and $\Delta_{2}(t)$ calculated for the vibration frequency $\Omega_{0}$ of $200 \mathrm{rad} / \mathrm{s}$ and the damping ratio $\eta$ of 0.01 are indicated by dashed-dotted lines. From these figures it follows that for the extended discrete signal an inexactness produced by the Hilbert transform method becomes a dominant reason of errors in amplitude and frequency predictions in the middle of the time interval, whereas an error generated by the end effect is prevailing only in close proximity of its upper limit.

\section{Conclusions}

The Hilbert transform has been recognized as a useful tool for an identification of instantaneous signal parameters in different branches of science and technology. In the paper, some of issues encountered when applying the discrete Hilbert transform to a reconstruction of amplitude and frequency of vibration signals have been discussed. The problem was examined via numerical simulations carried out for sinusoidal damped vibrations often encountered in engineering practice.

An inexactness of the Hilbert transform method was modelled by additive and multiplicative approaches which showed that the error produced by a numerical algorithm generates fictitious amplitude and frequency oscillations. Calculation results have demonstrated that for a small value of the damping ratio, these oscillations are substantial at both ends of a discrete signal. Thus, the end effect resulting from a finite signal duration influences a quality of a reconstruction of signal parameters even at a small damping. Huge erroneous amplitude and frequency oscillations noted for a large damping cause the Hilbert transform method to be totally unusable for fast decaying vibration signals.

In order to improve an accuracy of the Hilbert transform method, a numerical algorithm consisting in the appropriate modification of a discrete signal, was proposed. Although this algorithm is simple and easy to implement, for a small damping it visibly decreased the end error at the lower limit of time interval and more importantly, it greatly suppressed amplitude and frequency errors for strongly damped vibrations. As was found, in this case for the extended discrete signal, a main cause of the error is that for the product of exponential and sinusoidal functions the Bedrosian identity is not satisfied.

\section{References}

[1] D. Gabor, J. Inst. Electr. Eng. 93, 429 (1946).

[2] M. Feldman, J. Sound Vibrat. 295, 518 (2006).

[3] Y. Yan, K. Ahmad, M. Kunduk, D. Bless, J. Voice 19, $161(2005)$

[4] M. Meissner, Arch. Acoust. 34, 491 (2009).

[5] M. Meissner, Acta Phys. Pol. A 119, 1031 (2011).

[6] M. Meissner, Cent. Eur. J. Phys. 5, 293 (2007).

[7] M. Meissner, Arch. Acoust. 33, 159 (2008).

[8] M. Meissner, Acta Phys. Pol. A 118, 123 (2010).

[9] E. Bedrosian, Proc. IEEE 51, 868 (1963).

[10] L. Huageng, F. Xingjie, E. Bugra, Am. Inst. Aero. Astr. J. 47, 923 (2009).

[11] S.L. Hahn, The Hilbert Transforms in Signal Processing, Artech House Inc., Boston 1996.

[12] S. Kak, Proc. IEEE 58, 585 (1970).

[13] M. Meissner, Appl. Acoust. 69, 583 (2008).

[14] W.T. Thomson, Theory of Vibration with Applications, 4th ed., Prentice Hall, New Jersey 1993. 